\title{
The chemical composition of Amazonas plants ${ }^{(*)}$
}

\section{A Catalogue, edited by Setor de Fitoquímica, INPA, Manaus, Amazonas}

FAMILY :

Guttiferae

OCURRENCE : Manaus, Amazonas

TRUNK WOOD :

Lorostemin (I)

REFERENCE :

Braz Filho, R., Coutinho Lemos, M. de J. and Gottlieb, O. R. (1973) Phytochemistry 12, 947.

\author{
SPECIES : \\ Lorostemon coelhoi Paula \\ L. negrensis Froes
}

(I)

SPECIE :

Aniba canelilla (H.B.K.) Mez

"Casca Preciosa"

Ocurrence : Manaus, Amazonas.

TRUNK WOOD :

O-Methyleugenol 1

Eugenol 1

1-Nitro-2-phenylethane 1

6- (3', 4'-Dimethoxy-trans-styryl) -4-methoxy-2-pyrone 2

REFERENCES :

1. Gottlieb, O. R. and Taveira Magalhães, M. (1959) J. Org. Chem. 24, 2070.

2. Andrade da Mata Rezende, C. M., von Bülow,

M. V., Gottlieb. O. R. Lamêgo Vieira Pinho,

S. and da Rocha, A. I. (1971) Phytochemistry,<smiles>COc1cc(/C=C/c2ccc(OC)c(OC)c2)oc(=O)c1</smiles>

(I) 10,3167 .

(*) - Contributions to this catalogue, which will be continued in subsequent issues of this Journal, are invited, and should be submitted to adress given above. 
FAMILY :

Laúraceae

OCURRENCE : Amazônia
SPECTES :

Aniba duckei Kost.

"pau rosa"

TRUNK WOOD :

4-Methoxyphenylcoumalin (4-methoxy-6-phenyl-2-pyrone) 4

4-Metroxyparacotoin (4-methoxy-6-piperonyl-2-pyrone) 3

Anibine [4-methoxy-6-(3-pyridyl)-2-pyrone] 3

Cotoin (2,6-dihydroxy-4-methoxy-benzophenone)

Linalool 1,2

1,8-Cineol 1,2

$\propto$-Terpineol 1,2

$\propto$-Pinene and other terpenes 1,2

REFERENCES :

1. Guenther, E. (1963) "The Essential Oils" V. II,

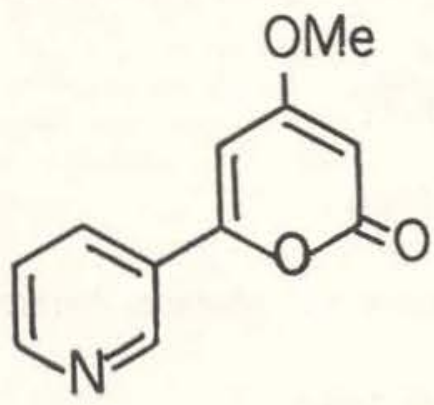

(I) p. $54,167,188,708$.

2. Fineberg, M., Gottlieb, O. R., Lima Guimarães, M., Maravalhas, N. and Taveira Magalhães, M . (1964) Perfumery \& Essencial Oil Record 55, 253.

3. Gottlieb, O. R., Mors, W. B. and Djerassi, C. (1957) J. Am. Chem. Soc. 79, 4507.

4. Gottlieb, O. R., Taveira Magalhães, M. and Mors, W. B. (1960) Anais Assoc. brasil, Quim. 19, 193.

5. Gottlieb, O. R. and Mors, W. B. (1958) J. Am. Chem. Soc. 80, 2263.

FAMILY :

Lauraceae

OCurrence : Amazônia

TRUNK wOOD :

4-Methoxyparacotoin (4-methoxy-6-piperonyl-2-pyrone) 3

Linalool and terpenes 1

Anibine [4-methoxy-6-(3-pyridyl)

-2-pyrone] 3 (I)

Sitosterol 3

Pinocembrin 2 (II)

Rubranine 4 (III)

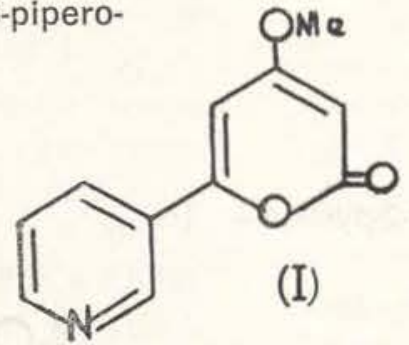

\section{Species :}

Aniba rosaedora Ducke

"pau rosa"

\section{REFERENCES :}

1. Guenther, E. (1963) "The Essential Oils" V. II, 167.

2. Gottlieb, O. R. and Mors, W. B. (1958) J. Am. Chem. Soc. 80, 2263.

3. Gottlieb, O. R., Taveira Magalhães, M. and Mors, W. B. (1960) Anais Assoc. brasil. Quim. 19, 193.

4. Combes, G., Vassort, P. L. and Winternitz, F. (1970) Tetrahedron 26, 5981.

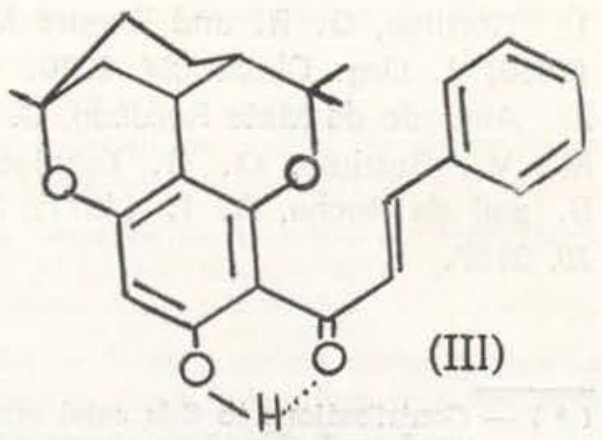

